\title{
Cause-specific death in hospitalized individuals infected with SARS-CoV-2: More than just acute respiratory failure or thromboembolic events.
}

\section{Cobos-Siles Marta}

Department of Internal Medicine, Hospital Universitario Río Hortega, Valladolid, Spain.

\section{Cubero-Morais, Pablo}

Department of Internal Medicine, Hospital Universitario Río Hortega, Valladolid, Spain.

Arroyo-Jiménez, Irene

Department of Internal Medicine, Hospital Universitario Río Hortega, Valladolid, Spain.

Hernández-Gómez, Laura

Department of Internal Medicine, Hospital Universitario Río Hortega, Valladolid, Spain.

\section{Vargas-Parra, Derly Judith}

Department of Internal Medicine, Hospital Universitario Río Hortega, Valladolid, Spain.

\section{González-Fernández, María}

Department of Internal Medicine, Hospital Universitario Río Hortega, Valladolid, Spain.

\section{Cazorla-González, Marina}

Department of Internal Medicine, Hospital Universitario Río Hortega, Valladolid, Spain.

Gabella-Martín, Miriam

Department of Internal Medicine, Hospital Universitario Río Hortega, Valladolid, Spain.

Ruíz-Albi, Tomás

Department of Pneumology, Hospital Universitario Río Hortega, Valladolid, Spain.

Berezo-García, José Angel

Department of Critical Care Medicine, Hospital Universitario Río Hortega, Valladolid, Spain.

\section{García-Cruces-Méndez, Jesús Fernando}

Department of Preventive Medicine \& Hospital Epidemiology, Hospital Universitario Río Hortega, Valladolid, Spain.

\section{Miramontes-González, Pablo}

Department of Internal Medicine, Hospital Universitario Río Hortega, Valladolid, Spain.

Corral-Gudino, Luis ( $\square$ lcorral@saludcastillayleon.es )

Department of Internal Medicine, Hospital Universitario Río Hortega, Valladolid, Spain. https://orcid.org/0000-0003-0151-5420 
Keywords: COVID-19; cause of death; age distribution; risk factor; frail elderly: hospital mortality

Posted Date: July 6th, 2020

DOI: https://doi.org/10.21203/rs.3.rs-39922/v1

License: (c) (1) This work is licensed under a Creative Commons Attribution 4.0 International License. Read Full License

Version of Record: A version of this preprint was published at Internal and Emergency Medicine on July 6th, 2020. See the published version at https://doi.org/10.1007/s11739-020-02485-y. 


\section{Abstract}

Background: Infection with SARS-CoV-2 is becoming the leading cause of death in most countries during the 2020 pandemic.

Objective: to assess the association between COVID-19 and cause-specific death.

Design: Retrospective cohort study.

Setting and Participants: We included data from inpatients diagnosed with COVID-19 between March 18 and April 21, 2020, who died during their hospital stay. Demographic, clinical and management data were collected. Causes of death were ascertained by review of medical records.

Results: The sample included 100 individuals. The median age was 85 (IQR 15), 59\% were men. Fifteen patients (15\%) died from complications unrelated to COVID-19. In univariate analysis, these patients compared to those who died from COVID-19 complications had a greater likelihood of dementia (47\% vs $20 \%$ ) and dependency in activities of daily living ( $93 \%$ vs $48 \%$ ), higher Charlson comorbidity index scores (6 vs 5), a lower likelihood of fever at diagnosis ( $47 \%$ vs $77 \%$ ), and higher lymphocyte counts (1,000 vs 660). In multivariate analysis, patients were older (OR 1.10, 95\% $\mathrm{Cl} 1.00-1.23$ ) and more often had heart failure (OR 5.58, 95\% Cl 1.09-28.66). The presence of X-ray infiltrates was uncommon (OR $0.03,95 \% \mathrm{Cl}$ $0.01-0.17)$. A higher percentage of patient deaths from causes unrelated to COVID-19 complications occurred during the two first weeks of the pandemic.

Conclusions: Fifteen percent of patients with COVID-19 died from causes unrelated to COVID-19 complications. Most of these patients had more comorbidities and were frail and elderly. These findings can partially explain the excess mortality in older people.

\section{Highlights}

- The case fatality rate of patients with SARS-CoV-2 infection is higher in older patients.

- Neither an excessive immune response nor the development of ARDS in severe COVID-19 disease fully explain the excess mortality in older people.

- Nearly $15 \%$ of patients with SARS-CoV-2 died without developing ARDS or other complications directly associated with the viral infection.

- Frailty, dependency for activities of daily living, and heart failure were associated with death from other causes than the viral infection.

- The more overwhelmed with COVID-19 hospitals are, the higher the proportion of COVID-19 patients dying of causes unrelated to the viral infection.

\section{Introduction}


The first case of coronavirus disease 2019 (COVID-19) caused by severe acute respiratory syndrome coronavirus 2 (SARS-CoV-2) was identified in China in December 2019. Since then, increasing numbers of deaths have been related to SARS-CoV-2. The case fatality rate across countries and territories varies from less than $1 \%$ to over $10 \%$ [1]. These rates should be interpreted with caution, given the many variations and the number of factors influencing the rate $[2,3]$.

Many risk factors are associated with death in COVID-19 infection, with old age a common factor [4]. Case fatality rates ranged from $1.3 \%$ in patients $<70$ years old to $8.0 \%$ in those $70-79$ years old and $14.8 \%$ in patients aged $>80$ years in a large Chinese series [5]. This is also true for critical cases, with a case fatality rate of $15 \%$ in patients $\leq 63$ years old admitted to intensive care units vs $36 \%$ in patients $>63$ years [6]. Underlying health conditions such as respiratory disease (odds ratio [OR] 2.46, 95\% confidence interval [Cl] 1.76-3.44), cardiovascular diseases (OR 3.42, 95\% Cl 1.88-6.22) and hypertension (OR 2.36, $95 \% \mathrm{Cl} 1.46$ to 3,83 ) have also been associated with increased death risk [7], as have been obesity, smoking and male sex $[8,9]$.

The main complications associated with SARS-CoV-2 infection and death are acute respiratory distress syndrome (ARDS), disseminate intravascular coagulation and sepsis [10]. An excessive immune response causing a cytokine storm is involved in many cases where the disease progresses rapidly [11]. These acute complications due to the infection itself could explain the death rate in young people but leave open the question of the extremely high mortality in older people, especially in very old or frail people. This excess mortality in the oldest patients could partly explain the differences in death rates between countries and regions, with higher mortality in countries such as Spain and Italy, which have some of the highest life expectancies worldwide [12].

This case series describes the cause of death in patients infected with SARS-CoV-2 in a single hospital. We aimed to differentiate the percentage of deaths directly associated with the infection and its complications (lung injury or thromboembolic events) and those where the illness acted as a trigger to decompensate previous comorbid conditions, with this decompensation being the cause of death.

\section{Materials And Methods}

\section{Study population}

This is a retrospective cohort study of all patients diagnosed with COVID-19 infection who died during their hospitalization at Hospital Universitario Río Hortega, Valladolid, Spain, from 18 March to 21 April 2020. The hospital ethics committee approved this study, and due to the nature of the retrospective chart review, waived the need for informed consent from individual patients.

Patients were included if they were diagnosed with confirmed COVID-19 and died during the study period. Patients were diagnosed according to World Health Organization guidance [13]. Laboratory confirmation for SARS-CoV-2 was defined as a positive result of real-time reverse transcriptase polymerase chain reaction (RT-PCR) assay of nasal and pharyngeal swabs or a positive serology test. 


\section{Data collection}

A trained team of internal medicine physicians and specialist registrars reviewed and collected epidemiological, clinical and outcome data from electronic medical records. Patient confidentiality was protected by deidentification.

Every patient's medical record was independently assessed by one of the researchers (MCS, MGM, PCM, IAJ, MRH, LHG, DVP, MCG, MGF). When the cause of death was unclear, the medical record was assessed by a second researcher. Differences in opinion were resolved by discussion and consensus or by consulting one of the senior authors (LCG) if needed.

\section{Study outcomes and definitions}

The primary outcome was the definition of the cause of death of the patients. As the secondary outcome, we assessed the development of ARDS, hyperinflammation response, sepsis or thromboembolic events.

Acute respiratory distress syndrome (ARDS) was identified according to the Berlin Definition [14]. All the following criteria were required:

1. Onset: Respiratory symptoms must begin within one week of a known clinical insult, or the patient must have new or worsening symptoms during the past week.

2. Chest imaging: Bilateral opacities must be present on a chest radiograph or computed tomographic (CT) s These opacities must not be fully explained by pleural effusions, lobar collapse, lung collapse or pulmonary nodules.

3. Origin of pulmonary infiltrates: The patient's respiratory failure must not be fully explained by cardiac failure or fluid overload. ARDS can be diagnosed once cardiogenic pulmonary oedema and alternative causes of acute hypoxemic respiratory failure have been excluded.

4. Oxygenation impairment: A moderate to severe impairment of oxygenation must be present, as defined by the ratio of arterial oxygen tension to fraction of inspired oxygen $\left(\mathrm{PaO}_{2} / \mathrm{FiO}_{2}\right)$, or the ratio of oxygen saturation to $\mathrm{FiO}_{2}\left(\mathrm{SaO}_{2} / \mathrm{FiO}_{2}\right)$ when $\mathrm{PaO}_{2}$ is not available. The severity of the hypoxemia defines the severity of ARDS:

a. Mild: $\mathrm{PaO}_{2} / \mathrm{FiO}_{2} 200-300 \mathrm{mmHg}$ or $\mathrm{SaO}_{2} / \mathrm{FiO}_{2} 237-317 \mathrm{mmHg}$

b. Moderate: $\mathrm{PaO}_{2} / \mathrm{FiO}_{2} 100-200 \mathrm{mmHg}$ or $\mathrm{SaO}_{2} / \mathrm{FiO}_{2} 155-237 \mathrm{mmHg}$

c. Severe: $\mathrm{PaO}_{2} / \mathrm{FiO}_{2} \leq 100 \mathrm{mmHg}$ or $\mathrm{SaO}_{2} / \mathrm{FiO}_{2}<155 \mathrm{mmHg}$.

Hyperinflammation response: We considered a hyperinflammatory response present in the context of moderate to severe COVID-19 infection with a lymphocyte count of $<1,000 \times 10^{9} / \mathrm{mL}$, and one of the following laboratory parameters:

1. Serum ferritin $>1,000 \mathrm{ng} / \mathrm{mL}$ 
2. C-reactive protein $>150 \mathrm{mg} / \mathrm{L}$

3. D-dimer $>800 \mu \mathrm{g} / \mathrm{L}$

4. Interleukin $6>20 \mathrm{pg} / \mathrm{mL}$.

Cause-specific death in individuals infected with SARS-CoV-2: We defined clinical criteria for assessing COVID-related causes of death. It was not possible to support the diagnosis of death by necropsies, as they were not available.

We considered that death was directly caused by SARS-CoV-2 infection complications when the patient developed:

1. ARDS or hyperinflammation response that led to their death.

2. Acute respiratory failure leading to death without meeting ARDS and hyperinflammation response criteria, and severe lung injury likely due to COVID-19 on X-ray or CT.

3. Acute respiratory failure or thrombotic events in the context of pulmonary embolism or other forms of venous thromboembolism that led to death.

4. Septic shock due to SARS-CoV-2 that led to their death.

We considered that the cause of death was unrelated to complications of SARS-CoV-2 infection when:

1. The patient developed acute respiratory failure caused by cardiogenic pulmonary oedema or other alternative causes of acute hypoxemic respiratory failure unrelated to COVID-19 that led to death. There were no lung injuries on X-ray or CT likely caused by COVID-19, or they were mild.

2. The patient developed multiorgan failure associated with frailty that led to death. Septic shock was excluded. There were no lung injuries on X-ray or CT likely caused by COVID-19, or they were mild to moderate.

3. The patient's death was from any confirmed cause unrelated to COVID-19 complications, including septic shock due to another microorganism.

\section{Statistical analysis}

The sample size was equal to the number of patients who died during the study period. Descriptive analysis of the continuous variables is expressed as the median and interquartile range (IQR). Categorical variables are presented as the number of patients (\%) with $95 \% \mathrm{Cl}$.

Differences in distributions of patient characteristics by outcome subgroup were reported using differences with $95 \% \mathrm{Cls}$. Categorical data were compared using the Chi square test or the Fisher exact test when needed. For crosstabs larger than $2 \times 2$, we used the Linear-by-Linear Association test to assess for trends. Continuous data were compared using Student's t-test or the Mann-Whitney rank sum test with non-parametric variables. To assess the associations of the different variables with the cause of death, we performed backwards stepwise logistic regression. ORs are presented with $95 \% \mathrm{Cls}$. 
All tests were two-sided with statistical significance set at $p<0.05$. All analyses were performed with SPSS, version 21.0 (IBM SPSS).

\section{Results}

A total of 734 patients referred to our hospital were diagnosed as infected with SARS-CoV-2 during the study period. Of these, 656 (89\%, 95\% Cl 87-91\%) patients were admitted to hospital (median age 71 years, IQR $29 ; 52 \%$ were male). Seventy-eight patients were discharged on the same day directly from the emergency room because they had mild disease without pneumonia. One hundred patients died $(15.2 \%$, $95 \% \mathrm{Cl} 13-18 \%)$. The distributions of inpatients and the mortality rate according to age group are detailed in Figure 1. At the time of writing, $17 \%$ of the patients admitted during the study period were still in hospital.

The median age of the patients who died was 85 years (IQR 15), and $59 \%$ were male. Basal characteristics, comorbidities and chronic treatment are detailed in Table 1. In 15 patients, the cause of death was attributed to other causes than the own viral infection. These patients were older, with more long-term conditions and higher Charlson comorbidity scores, and more frequently had heart failure, dementia or cerebrovascular disease. They had a higher grade of dependency for activities of daily living. More frequently, the X-ray was normal and no fever was present at admission.

Table 1. Basal characteristics. 


\begin{tabular}{|c|c|c|c|c|}
\hline Characteristics & $\begin{array}{l}\text { Total } \\
(\mathrm{n}= \\
100)\end{array}$ & $\begin{array}{c}\text { Deaths caused directly } \\
\text { by SARS-CoV-2 infection } \\
(\mathrm{n}=85)\end{array}$ & $\begin{array}{l}\text { Deaths unrelated to } \\
\text { SARS-CoV-2 } \\
\text { complications }(\mathrm{n}=15)\end{array}$ & $\mathrm{p}$ \\
\hline $\begin{array}{l}\text { Age, median (IQR), } \\
\text { years }\end{array}$ & $\begin{array}{l}85 \\
(15)\end{array}$ & $79(16)$ & $89(6)$ & 0.010 \\
\hline $\begin{array}{l}<50 \\
50-59 \\
60-69 \\
70-79 \\
80-89 \\
\geq 90\end{array}$ & $\begin{array}{c}2 \\
(2 \%) \\
2 \\
(2 \%) \\
9 \\
(9 \%) \\
31 \\
(31 \%) \\
32 \\
(32 \%) \\
24 \\
(24 \%)\end{array}$ & $\begin{array}{c}2(2 \%) \\
2(2 \%) \\
9(11 \%) \\
30(35 \%) \\
24(29 \%) \\
18(22 \%)\end{array}$ & $\begin{array}{cc}0 & (0 \%) \\
0 & (0 \%) \\
0 & (0 \%) \\
1 & (7 \%) \\
8(44 \%) \\
6(33 \%)\end{array}$ & $0.007^{*}$ \\
\hline Males & $\begin{array}{c}59 \\
(59 \%)\end{array}$ & $53(62 \%)$ & $6(40 \%)$ & 0.105 \\
\hline $\begin{array}{l}\text { Place of residence } \\
\text { Home } \\
\text { Nursing home }\end{array}$ & $\begin{array}{c}57 \\
(57 \%) \\
43 \\
(43 \%)\end{array}$ & $\begin{array}{l}55(62 \%) \\
32(38 \%)\end{array}$ & $\begin{array}{c}4(27 \%) \\
11(73 \%)\end{array}$ & 0.010 \\
\hline $\begin{array}{l}\text { Comorbidities } \\
\text { Hypertension } \\
\text { Dyslipidaemia } \\
\text { Myocardial infarction } \\
\text { Heart failure } \\
\text { Cerebrovascular } \\
\text { disease } \\
\text { Dementia } \\
\text { COPD } \\
\text { Asthma } \\
\text { SAHS } \\
\text { Interstitial lung } \\
\text { disease } \\
\text { Connective tissue } \\
\text { disease } \\
\text { Peptic ulcer disease } \\
\text { Diabetes mellitus } \\
\text { Uncomplicated } \\
\text { End organ damage } \\
\text { Chronic liver disease } \\
\text { Mild } \\
\text { Moderate or severe } \\
\text { Chronic kidney } \\
\text { disease } \\
\text { Solid tumour } \\
\text { Localized } \\
\text { Metastatic } \\
\text { Leukaemia or } \\
\text { lymphoma Current or }\end{array}$ & $\begin{array}{c}71 \\
(71 \%) \\
42 \\
(42 \%) \\
16 \\
(16 \%) \\
19 \\
(19 \%) \\
12 \\
(12 \%) \\
24 \\
(24 \%) \\
6 \\
(6 \%) \\
4 \\
(4 \%) \\
6 \\
(6 \%) \\
1 \\
(1 \%) \\
0 \\
(0 \%) \\
12 \\
(12 \%) \\
\\
25 \\
(25 \%)\end{array}$ & $\begin{aligned} 58 & (68 \%) \\
37 & (44 \%) \\
14 & (17 \%) \\
13 & (15 \%) \\
7 & (8 \%) \\
17 & (20 \%) \\
5 & (6 \%) \\
3 & (4 \%) \\
4 & (5 \%) \\
1 & (1 \%) \\
0 & (0 \%) \\
10 & (12 \%) \\
& 1(25 \%) \\
3 & (4 \%) \\
& \\
4 & (5 \%) \\
2 & (2 \%) \\
14 & (17 \%) \\
& \\
12 & (14 \%) \\
2 & (2 \%) \\
4 & (5 \%) \\
18 & (21 \%)\end{aligned}$ & $\begin{array}{c}13(87 \%) \\
5(33 \%) \\
2(13 \%) \\
6(40 \%) \\
5(33 \%) \\
7(47 \%) \\
1(7 \%) \\
1(7 \%) \\
2(13 \%) \\
0(0 \%) \\
1(7 \%) \\
2(13 \%) \\
4(27 \%) \\
1(7 \%) \\
0(0 \%) \\
0(0 \%) \\
2(13 \%) \\
0(0 \%) \\
0(0 \%) \\
1(7 \%) \\
3(20 \%)\end{array}$ & $\begin{array}{l}0.219 \\
0.461 \\
1.000 \\
\mathbf{0 . 0 3 6} \\
\mathbf{0 . 0 1 6} \\
\mathbf{0 . 0 4 6} \\
0.484 \\
0.558 \\
0.220 \\
1.000 \\
0.150 \\
1.000 \\
\\
1.000 \\
0.484 \\
\\
1.000 \\
1.000 \\
1.000 \\
\\
0.204 \\
1.000 \\
0.564 \\
1.000\end{array}$ \\
\hline
\end{tabular}




\begin{tabular}{|c|c|c|c|c|}
\hline past smoker & $\begin{array}{c}4 \\
(4 \%) \\
4 \\
(4 \%) \\
2 \\
(2 \%) \\
16 \\
(16 \%) \\
12 \\
(12 \%) \\
2 \\
(2 \%) \\
5 \\
(5 \%) \\
21 \\
(21 \%)\end{array}$ & & & \\
\hline $\begin{array}{l}\text { Number of } \begin{array}{l}\text { long-term } \\
\text { conditions, } \\
\text { (IQR) }\end{array} \\
\end{array}$ & $3(3)$ & $3(4)$ & $5(4)$ & 0.064 \\
\hline $\begin{array}{l}\text { Charlson comorbidity } \\
\text { index, median (IQR) }\end{array}$ & $5(3)$ & $5(2)$ & $6(2)$ & 0.040 \\
\hline $\begin{array}{l}\text { Performance in } \\
\text { activities of daily living } \\
\text { Independent } \\
\text { Slight dependency } \\
\text { Moderate } \\
\text { dependency } \\
\text { Severe dependency } \\
\text { Total dependency }\end{array}$ & $\begin{array}{c}46 \\
(46 \%) \\
21 \\
(21 \%) \\
14 \\
(14 \%) \\
8 \\
(8 \%) \\
11 \\
(11 \%)\end{array}$ & $\begin{array}{c}45(52 \%) \\
18(21 \%) \\
10(12 \%) \\
7(8 \%) \\
5(6 \%)\end{array}$ & $\begin{array}{c}1(7 \%) \\
3(20 \%) \\
4(27 \%) \\
1(7 \%) \\
6(40 \%)\end{array}$ & 0.000 \\
\hline $\begin{array}{l}\text { Chronic treatment } \\
\text { ACEs } \\
\text { ARBs } \\
\text { Statins } \\
\text { Metformin } \\
\text { IDPP-4 } \\
\text { Insulin } \\
\text { Inhaled } \\
\text { corticosteroids } \\
\text { Oral corticosteroids } \\
\text { Immunomodulators }\end{array}$ & $\begin{array}{c}25 \\
(25 \%) \\
25 \\
(25 \%) \\
32 \\
(32 \%) \\
18 \\
(18 \%) \\
11 \\
(11 \%) \\
6 \\
(6 \%) \\
9 \\
(9 \%) \\
3 \\
(3 \%)\end{array}$ & $\begin{array}{c}18(21 \%) \\
20(24 \%) \\
29(34 \%) \\
13(15 \%) \\
10(12 \%) \\
6(7 \%) \\
8(9 \%) \\
3(4 \%) \\
4(5 \%)\end{array}$ & $\begin{array}{cc}7 & (47 \%) \\
5 & (33 \%) \\
3 & (20 \%) \\
5 & (33 \%) \\
1 & (7 \%) \\
0 & (0 \%) \\
1 & (7 \%) \\
0 & (0 \%) \\
0 & (0 \%)\end{array}$ & $\begin{array}{l}0.051 \\
0.518 \\
0.375 \\
0.138 \\
1.000 \\
0.587 \\
1.000 \\
1.000 \\
1.000\end{array}$ \\
\hline
\end{tabular}


ACEs: angiotensin-converting enzyme inhibitors, ARBs: angiotensin II receptor blockers, COPD: chronic obstructive pulmonary disease, RT-PCR: reverse transcription polymerase chain reaction, SAHS: sleep apnoea-hypopnea syndrome.

Among the 15 patients whose deaths were not directly caused by SARS-CoV-2 infection, eight very frail patients (53\%) died without developing any SARS-CoV-2 complication, with the cause of death attributed to wasting associated with age; two (13\%) died of sepsis caused by bacterial infection; 2 (11\%) died of heart failure or pulmonary oedema; two (13\%) died of kidney failure; and one (7\%) died of bronchoaspiration.

The most commonly reported symptoms are detailed in Table 2. Patients who died of COVID-19 complications were more frequently feverish or had fever at admission. Dyspnoea was the most common symptom in both groups. Nearly half of the patients who died without COVID-19 complications had normal X-rays, and lymphocyte and white blood cell counts were higher in these patients.

Table 2. Initial clinical, radiological and laboratory parameters and specific COVID-19 treatment. 


\begin{tabular}{|c|c|c|c|c|}
\hline Characteristics & $\begin{array}{l}\text { Total } \\
(\mathrm{n}= \\
100)\end{array}$ & $\begin{array}{c}\text { Deaths caused } \\
\text { directly by SARS-CoV- } \\
2 \text { infection }(n=85)\end{array}$ & $\begin{array}{l}\text { Deaths unrelated to } \\
\text { SARS-CoV-2 } \\
\text { complications }(\mathrm{n}= \\
15)\end{array}$ & $\mathrm{p}$ \\
\hline $\begin{array}{l}\text { Days from illness onset to } \\
\text { hospital admission, median } \\
\text { (IQR), days }\end{array}$ & $4(5)$ & $5(5)$ & $3(6)$ & 0.404 \\
\hline $\begin{array}{l}\text { Signs and symptoms at } \\
\text { diagnosis } \\
\text { Shortness of breath } \\
\text { Fever } \\
\text { Cough } \\
\text { Diarrhoea or vomiting } \\
\text { Abdominal pain } \\
\text { Anosmia or ageusia } \\
\text { Asthenia } \\
\text { Arthromyalgia } \\
\text { Sore throat } \\
\text { Headache }\end{array}$ & $\begin{array}{c}78 \\
(78 \%) \\
72 \\
(72 \%) \\
45 \\
(45 \%) \\
12 \\
(12 \%) \\
5(5 \%) \\
20 \\
(20 \%) \\
13 \\
(13 \%) \\
3(3 \%) \\
3(3 \%) \\
13 \\
(13 \%)\end{array}$ & $\begin{aligned} 64 & (75 \%) \\
65 & (77 \%) \\
41 & (48 \%) \\
12 & (14 \%) \\
5 & (6 \%) \\
18 & (22 \%) \\
11 & (13 \%) \\
3 & (4 \%) \\
3 & (4 \%) \\
11 & (13 \%)\end{aligned}$ & $\begin{array}{c}14(93 \%) \\
7(47 \%) \\
4(27 \%) \\
0(0 \%) \\
0(0 \%) \\
2(11 \%) \\
2(11 \%) \\
0(0 \%) \\
0(0 \%) \\
2(11 \%)\end{array}$ & $\begin{array}{l}0.180 \\
0.028 \\
0.122 \\
0.204 \\
1.000 \\
0.298 \\
1.000 \\
1.000 \\
1.000 \\
1.000\end{array}$ \\
\hline $\begin{array}{l}\text { X-ray/CT scan findings } \\
\text { Normal } \\
\text { Unilateral infiltrates } \\
\text { Bilateral infiltrates }\end{array}$ & $\begin{array}{c}14 \\
(14 \%) \\
16 \\
(16 \%) \\
70 \\
(70 \%)\end{array}$ & $\begin{aligned} 5 & (6 \%) \\
14 & (17 \%) \\
6 & (77 \%)\end{aligned}$ & $\begin{array}{l}9(60 \%) \\
2(13 \%) \\
5(33 \%)\end{array}$ & 0.000 \\
\hline $\begin{array}{l}\text { Diagnosis } \\
\text { RT-PCR positive } \\
\text { Ig test positive } \\
\text { Presumptive diagnosis }\end{array}$ & $\begin{array}{c}95 \\
(95 \%) \\
2(2 \%) \\
3(3 \%) \\
\end{array}$ & $\begin{aligned} 78 & (95 \%) \\
2 & (2 \%) \\
2 & (2 \%)\end{aligned}$ & $\begin{aligned} 14 & (93 \%) \\
0 & (0 \%) \\
1 & (7 \%)\end{aligned}$ & 0.289 \\
\hline $\begin{array}{l}\text { Laboratory parameters, } \\
\text { median (IQR) } \\
\text { SaFi } \\
\text { PaFi } \\
\text { White blood cells, } \mathrm{x} \\
10^{9} / \mathrm{mL} \\
\text { Lymphocytes, } \mathrm{x} 10^{9} / \mathrm{mL} \\
\text { Platelets, } \mathrm{x} 10^{9} / \mathrm{mL} \\
\text { Haemoglobin, } \mathrm{g} / \mathrm{dL} \\
\text { D-dimer, } \mathrm{mg} / \mathrm{mL} \\
\text { CRP, mg } / \mathrm{L} \\
\text { Serum ferritin, } \mathrm{ng} / \mathrm{mL} \\
\text { Creatinine, } \mathrm{mg} / \mathrm{dL} \\
\text { Sodium } \\
\text { ALT, U } / \mathrm{l}\end{array}$ & $\begin{array}{c}393 \\
(127) \\
236 \\
(114) \\
6,600 \\
(5,575) \\
700 \\
(500) \\
171 \\
(115) \\
129 \\
(25) \\
652 \\
(929)\end{array}$ & $\begin{array}{c}395(126) \\
238(117) \\
6,500(5,550) \\
660(500) \\
168(112) \\
127(27) \\
639(912) \\
110(110) \\
563(1287) \\
1.12(0.8) \\
134(9) \\
29(22) \\
51(45) \\
425(186)\end{array}$ & $\begin{array}{c}390(230) \\
233(62) \\
7,900(5,000) \\
1,000(900) \\
196(81) \\
131(21) \\
889(1865) \\
104(170) \\
564(557) \\
1.14(1.3) \\
137(4) \\
20(39) \\
49(24) \\
399(152)\end{array}$ & $\begin{array}{l}0.754 \\
0.889 \\
0.132 \\
0.039 \\
0.512 \\
0.133 \\
0.037 \\
0.925 \\
0.572 \\
0.671 \\
0.340 \\
0.878 \\
0.689 \\
0.828\end{array}$ \\
\hline
\end{tabular}




\begin{tabular}{|c|c|c|c|c|}
\hline $\begin{array}{l}\text { AST, U/l } \\
\mathrm{LDH}, \mathrm{U} / \mathrm{l}\end{array}$ & $\begin{array}{c}108 \\
(125) \\
563 \\
(1257) \\
1.13 \\
(0.5) \\
135 \\
(10) \\
27(38) \\
51(32) \\
424 \\
(141)\end{array}$ & & & \\
\hline $\begin{array}{l}\text { Treatments during study } \\
\text { period } \\
\text { Antibiotic agent } \\
\text { Azithromycin } \\
\text { Lopinavir and ritonavir } \\
\text { Hydroxychloroquine } \\
\text { Interferon } \\
\text { Tocilizumab } \\
\text { Corticosteroids } \\
\text { Anticoagulants } \\
\text { LMWH prophylaxis } \\
\text { LMWH extended } \\
\text { prophylaxis } \\
\text { LMWH therapy } \\
\text { Oral anticoagulants } \\
\text { No anticoagulants }\end{array}$ & $\begin{array}{c}96 \\
(96 \%) \\
84 \\
(84 \%) \\
51 \\
(51 \%) \\
85 \\
(85 \%) \\
14 \\
(14 \%) \\
3(3 \%) \\
25 \\
(25 \%) \\
\\
64 \\
(64 \%) \\
4(4 \%) \\
15 \\
(15 \%) \\
1(1 \%) \\
16 \\
(16 \%)\end{array}$ & $\begin{aligned} 81 & (95 \%) \\
70 & (82 \%) \\
49 & (58 \%) \\
71 & (84 \%) \\
14 & (17 \%) \\
3 & (4 \%) \\
24 & (28 \%) \\
& \\
52 & (61 \%) \\
4 & (5 \%) \\
13 & (15 \%) \\
1 & (1 \%) \\
15 & (18 \%)\end{aligned}$ & $\begin{array}{c}15(100 \%) \\
14(93 \%) \\
2(13 \%) \\
14(93 \%) \\
0(0 \%) \\
0(0 \%) \\
1(7 \%) \\
12(80 \%) \\
0(0 \%) \\
2(13 \%) \\
0(0 \%) \\
1(7 \%)\end{array}$ & $\begin{array}{l}0.391 \\
0.285 \\
0.002 \\
0.457 \\
0.120 \\
1.000 \\
0.385 \\
0.630\end{array}$ \\
\hline $\begin{array}{l}\text { Respiratory support } \\
\text { High-flow oxygen } \\
\text { Non-invasive ventilation } \\
\text { Invasive mechanical } \\
\text { ventilation } \\
\text { ECMO }\end{array}$ & $\begin{array}{c}1(1 \%) \\
11 \\
(11 \%) \\
20 \\
(20 \%) \\
1(1 \%)\end{array}$ & $\begin{aligned} 1 & (1 \%) \\
11 & (13 \%) \\
20 & (24 \%) \\
1 & (1 \%)\end{aligned}$ & $\begin{array}{l}0(0 \%) \\
0(0 \%) \\
0(0 \%) \\
0(0 \%)\end{array}$ & $\begin{array}{l}1.000 \\
0.206 \\
0.037 \\
1.000\end{array}$ \\
\hline
\end{tabular}

ALT: alanine aminotransferase, AST: aspartate aminotransferase, CRP: C-reactive protein, ECMO: extracorporeal membrane oxygenation, LDH: lactate dehydrogenase, LMWH: low molecular weight heparin, PaFi: arterial partial pressure of oxygen/fraction of inspired oxygen, SaFi: pulse oximetric saturation/fraction of inspired oxygen.

The COVID-19 complications are detailed in Table 3. Nearly four in five patients who died with complications associated with SARS-CoV-2 infection developed ARDS or hyperinflammation. The first weeks, with more pressure on the hospital from the admission of new patients with COVID-19, had higher rates of deaths unrelated to SARS-CoV-2 complications. 
Table 3. COVID-19 complications.

\begin{tabular}{|c|c|c|c|c|}
\hline Characteristics & $\begin{array}{l}\text { Total } \\
(\mathrm{n}= \\
100)\end{array}$ & $\begin{array}{c}\text { Deaths caused directly } \\
\text { by SARS-CoV-2 infection } \\
(\mathrm{n}=85)\end{array}$ & $\begin{array}{l}\text { Deaths unrelated to } \\
\text { SARS-CoV-2 } \\
\text { complications }(\mathrm{n}=15)\end{array}$ & $p$ \\
\hline $\begin{array}{l}\text { Days in hospital until } \\
\text { death, median (IQR), } \\
\text { days }\end{array}$ & $6(7)$ & $6(8)$ & $5(8)$ & 0.148 \\
\hline ARDS & $\begin{array}{c}70 \\
(70 \%)\end{array}$ & $70(82 \%)$ & $0(0 \%)$ & 0.000 \\
\hline Hyperinflammation & $\begin{array}{c}72 \\
(72 \%)\end{array}$ & $66(78 \%)$ & $6(40 \%)$ & 0.003 \\
\hline Septic shock & $\begin{array}{c}10 \\
(10 \%)\end{array}$ & $10(12 \%)$ & $0(0 \%)$ & 0.351 \\
\hline Pulmonary embolism & $\begin{array}{c}2 \\
(2 \%)\end{array}$ & $2(2 \%)$ & $0(0 \%)$ & 1.000 \\
\hline $\begin{array}{l}\text { Place of death } \\
\text { Hospital ward } \\
\text { Intensive care unit }\end{array}$ & $\begin{array}{c}80 \\
(80 \%) \\
20 \\
(20 \%) \\
\end{array}$ & $\begin{array}{l}65(77 \%) \\
20(23 \%)\end{array}$ & $\begin{array}{c}185(100 \%) \\
0(0 \%)\end{array}$ & 0.037 \\
\hline $\begin{array}{l}\text { Week of admission } \\
\text { 18-24 March } \\
\text { 25-31 March } \\
\text { 1-7 April } \\
\text { 8-14 April } \\
\text { 15-21 April }\end{array}$ & $\begin{array}{c}7 \\
(7 \%) \\
32 \\
(32 \%) \\
27 \\
(27 \%) \\
21 \\
(21 \%) \\
13 \\
(12 \%) \\
\end{array}$ & $\begin{aligned} 5 & (6 \%) \\
26 & (31 \%) \\
23 & (27 \%) \\
18 & (21 \%) \\
13 & (15 \%)\end{aligned}$ & $\begin{array}{c}2(13 \%) \\
6(40 \%) \\
4(27 \%) \\
3(20 \%) \\
0(0 \%)\end{array}$ & $0.084^{*}$ \\
\hline
\end{tabular}

ARDS: acute respiratory distress syndrome.

The variables associated with the cause of death are shown in Table 4.

Table 4. Variables significantly associated with deaths unrelated to COVID-19 complications.

\begin{tabular}{|l|l|l|l|}
\hline Variable & Crude OR (95\% CI) & Adjusted OR (95\% CI) & $\mathrm{p}$ \\
\hline Age & $1.12(1.03-1.22)$ & $1.10(1.01-1.23)$ & 0.048 \\
\hline Heart failure & $3.69(1.12-12.14)$ & $5.58(1.09-28.66)$ & 0.039 \\
\hline X-ray infiltrates on activities of daily & $0.04(0.01-0.16)$ & $0.03(0.01-0.17)$ & 0.000 \\
\hline $\begin{array}{l}\text { Dependence } \\
\text { living }\end{array}$ & $125.19)$ & & \\
\hline Cerebrovascular disease & $5.58(1.48-20.92)$ & & \\
\hline Dementia & $3.45(1.10-10.84)$ & & \\
\hline Fever or feverish at admission & $0.27(0.09-0.84)$ & & \\
\hline
\end{tabular}

CI: confidence interval, ns: statistically not significant, OR: odds ratio.

The evolution during the five weeks of the proportion of deaths attributed directly to COVID-19 is detailed in Figure 2. The number of new hospital admissions and the total number of deaths are also shown. 


\section{Discussion}

In this cohort study, we have reported the clinical characteristics of patients with COVID-19 admitted to a hospital who died during hospitalization. We analysed the cause of death to differentiate patients whose death was directly associated with development of COVID-19 complications (such is ARDS or pulmonary embolism) from infected patients who died of causes unrelated to any of these complications. Nearly $20 \%$ of patients died because of complications unrelated to COVID-19 infection. Most of these were old and frail patients, with only mild COVID-19 according to laboratory and X-ray findings. To the best of our knowledge, this is the first study analysing the cause of death of patients with COVID-19 to differentiate patients who died without developing COVID-19 complications.

The case fatality rate in patients with COVID-19 varies enormously across studies, from only $1.4 \%$ in the initial studies of the general population [3] to $21.9 \%$ in cohort studies of hospitalized patients with confirmed COVID-19 pneumonia [15] and more than $26 \%$ in patients admitted to intensive care units [6]. The mortality rates have been re-estimated [2]. The $15.2 \%$ rate of our study agrees with the expected mortality of $15.2 \%$ outside of China.

In all the literature, older people have a higher risk of death. However, the median age of 63 years in the first series in China [3] is far from our median age of 85 years. The marked aging of the population in our area and the greater life expectancy in Spain compared to China explain these differences. The older age of these COVID-19 victims brings the question of whether they have died prematurely [16]. For most young victims, there is no debate about the years of life lost but about their life quality. For most older people, this is also true, as more than $80 \%$ of patients in our series died of causes directly related to COVID-19 and could have lived longer without the infection.

As might be expected, age, comorbidities, frailty and dependency were associated with other causes of death unrelated to COVID-19 complications. The ongoing acute severe conditions [17], infections[18] or adverse events associated with prescribed drugs [19] could have played a role in these patients' deaths. Heart failure was the only comorbidity that emerged in the multivariate logistic regression analysis. Likely, the work overload caused by the infection decompensated an already failing heart. Heart failure and dementia are two of the leading causes of death in older adults in Spain [20].

Differences existed in the drugs prescribed to the two groups. Lopinavir and ritonavir, tocilizumab, interferon and corticosteroids were practically not prescribed to the COVID-19 patients who died of unrelated causes. This is easy to explain, as these patients had only mild or no lung injury from SARSCoV-2.

Although it did not reach statistical significance $(p=0.084)$, one of the most interesting associations was between unrelated cause of death and the time of hospital admission (Figure 1). Unsurprisingly, most cases of unrelated cause of death occurred during the first two weeks of the epidemic in our area, when the hospital was in the process of organizing new healthcare procedures for COVID-19 patients and when the number of new admitted patients almost exceeded the hospital capacity (12 of 16 hospital wards 
were transformed into COVID-19 wards in only two weeks). The isolation in rooms of patients with dependency for activities of daily living, the low number of ward rounds compared with non-epidemic periods, the ban on relatives or friends accompanying the patients in their rooms, and the overload of hospitals and staff due to the high number of patients admitted every day may have played a negative role in the care of the patients admitted with COVID-19 infection. This negative effect was certainly magnified in frail patients, where the hospital care is sometimes much more important than the drugs intravenous fluids administered [21]. The way we should manage acute complications related to the disease should be different than the way we manage acute decompensation of comorbid conditions in patients with an acute viral infection with no severe disease. For many older patients with no severe COVID-19 disease, the health care, including stimulation, feeding or physical activity encouragement, as well as the management of their comorbidities, was the cornerstone of their treatment, more than the use of antivirals or immunomodulators.

Our study has several limitations. Firstly, the difficulty in assessing the final cause of death in some patients, due to the impossibility of performing necropsies, forces interpreting the findings with caution, as exploratory and descriptive. Secondly, the study was conducted at a single-centre hospital with a limited sample size. Thirdly, the study only included patients admitted to the hospital. Patients who died in their nursing homes were not included, which may lead to underestimating the real proportion of patients who died without developing COVID-19 complications. Fourthly, some cases had incomplete clinical records and laboratory testing. To minimize the impact of missing data, we have incorporated all the electronic databases and scanned handwritten documents. We believe that one of the strengths of our study derives from the inclusion of all the patients who died during the study period.

\section{Conclusion}

Older and frail patients are at greater risk of death when infected by SARS-CoV-2. Although most deaths were associated with complications due to the own infection, a proportion of patients died from unrelated causes. This partially explains the high fatality rate in the oldest groups of the population. Several factors may be involved in the excess mortality of older people. The overload of the health system and consequent failure of hospital care standards and the need to increase isolation measures could have played a role in raising this mortality rate in patients with special care needs during admission.

\section{Declarations}

\section{Acknowledgement}

We thank Professor Félix Del Campo-Matías (Universidad de Valladolid) for useful discussion and comments on the manuscript.

We dedicate this manuscript to all our patients and especially to all their relatives and friends. We could not let most of you accompany your loved ones in their last moments. Be assured that they were never 
alone, and they always felt your support and affection close, thanks to the health workers who took care of them during hospitalization.

\section{Declarations of interest:}

None

\section{Financial support:}

None.

\section{Author contributions:}

1. Substantial contributions to:

1. Conception and design (LCG)

2. Acquisition of data (MCS, MGM, PCM, IAJ, MRH, LHG, DVP, MCG, MGF)

3. Analysis and interpretation of data (LCG, MCS, MGM, PCM, IAJ, MRH, LHG, DVP, MCG, MGF, JPMG, TRA, JABC, JFGCM)

2. Drafting the article or revising it critically for important intellectual content (LCG, MCS, MGM, PCM, IAJ, MRH, LHG, DVP, MCG, MGF, JPMG, TRA, JABC, JFGCM)

3. Final revision of the version to be published (LCG, MCS, MGM, PCM, IAJ, MRH, LHG, DVP, MCG, MGF, JPMG, TRA, JABC, JFGCM).

\section{References}

1. Coronavirus Update (Live): 2,646,247 Cases and 184,352 Deaths from COVID-19 Virus Pandemic Worldometer. https://www.worldometers.info/coronavirus/. Accessed 23 Apr 2020

2. Baud D, Qi X, Nielsen-Saines K, et al (2020) Real estimates of mortality following COVID-19 infection. The Lancet Infectious Diseases 0: https://doi.org/10.1016/S1473-3099(20)30195-X

3. Guan W-J, Ni Z-Y, Hu Y, et al (2020) Clinical Characteristics of Coronavirus Disease 2019 in China. N Engl J Med 382:1708-1720. https://doi.org/10.1056/NEJMoa2002032

4. Jordan RE, Adab P, Cheng KK (2020) Covid-19: risk factors for severe disease and death. BMJ 368:m1198. https://doi.org/10.1136/bmj.m1198

5. Wu Z, McGoogan JM (2020) Characteristics of and Important Lessons From the Coronavirus Disease 2019 (COVID-19) Outbreak in China: Summary of a Report of 72314 Cases From the Chinese Center for Disease Control and Prevention. JAMA 323:1239-1242. https://doi.org/10.1001/jama.2020.2648

6. Grasselli G, Zangrillo A, Zanella A, et al (2020) Baseline Characteristics and Outcomes of 1591 Patients Infected With SARS-CoV-2 Admitted to ICUs of the Lombardy Region, Italy. JAMA 323:15741581. https://doi.org/10.1001/jama.2020.5394 
7. Yang J, Zheng Y, Gou X, et al (2020) Prevalence of comorbidities in the novel Wuhan coronavirus (COVID-19) infection: a systematic review and meta-analysis. Int J Infect Dis. https://doi.org/10.1016/j.ijid.2020.03.017

8. Wang D, Hu B, Hu C, et al (2020) Clinical Characteristics of 138 Hospitalized Patients With 2019 Novel Coronavirus-Infected Pneumonia in Wuhan, China. JAMA 323:1061-1069. https://doi.org/10.1001/jama.2020.1585

9. Livingston E, Bucher K (2020) Coronavirus Disease 2019 (COVID-19) in Italy. JAMA. https://doi.org/10.1001/jama.2020.4344

10. Martins-Filho PR, Tavares CSS, Santos VS (2020) Factors associated with mortality in patients with COVID-19. A quantitative evidence synthesis of clinical and laboratory data. European Journal of Internal Medicine 0: https://doi.org/10.1016/j.ejim.2020.04.043

11. Mehta P, McAuley DF, Brown M, et al (2020) COVID-19: consider cytokine storm syndromes and immunosuppression. Lancet 395:1033-1034. https://doi.org/10.1016/S0140-6736(20)30628-0

12. Kontis V, Bennett JE, Mathers CD, et al (2017) Future life expectancy in 35 industrialised countries: projections with a Bayesian model ensemble. Lancet 389:1323-1335. https://doi.org/10.1016/S0140-6736(16)32381-9

13. Clinical management of severe acute respiratory infection when COVID-19 is suspected. https://www.who.int/publications-detail/clinical-management-of-severe-acute-respiratory-infectionwhen-novel-coronavirus-(ncov)-infection-is-suspected. Accessed 2 Apr 2020

14. ARDS Definition Task Force, Ranieri VM, Rubenfeld GD, et al (2012) Acute respiratory distress syndrome: the Berlin Definition. JAMA 307:2526-2533. https://doi.org/10.1001/jama.2012.5669

15. Wu C, Chen X, Cai Y, et al (2020) Risk Factors Associated With Acute Respiratory Distress Syndrome and Death in Patients With Coronavirus Disease 2019 Pneumonia in Wuhan, China. JAMA Intern Med e200994. https://doi.org/10.1001/jamainternmed.2020.0994

16. Before their time - Would most covid-19 victims have died soon, without the virus? | Graphic detail | The Economist. https://www.economist.com/graphic-detail/2020/05/02/would-most-covid-19victims-have-died-soon-without-the-virus?utm_campaign=coronavirus-specialedition\&utm_medium=newsletter\&utm_source=salesforce-marketing-cloud\&utm_term=2020-0502\&utm_content=article-link-2https://www.msn.com/es-es/? cobrand=acer17 win10.msn.com\&ocid=ACERDHP17\&pc=ACTE. Accessed 2 May 2020

17. Mehra MR, Desai SS, Kuy S, et al (2020) Cardiovascular Disease, Drug Therapy, and Mortality in Covid-19. N Engl J Med. https://doi.org/10.1056/NEJMoa2007621

18. Wang CJ, Bair H, Yeh C-C (2020) How to Prevent and Manage Hospital-Based Infections During Coronavirus Outbreaks: Five Lessons from Taiwan. Journal of Hospital Medicine. https://doi.org/10.12788/jhm.3452

19. Bonow RO, Hernandez AF, Turakhia M (2020) Hydroxychloroquine, Coronavirus Disease 2019, and QT Prolongation. JAMA Cardiol. https://doi.org/10.1001/jamacardio.2020.1782 
20. Soriano JB, Rojas-Rueda D, Alonso J, et al (2018) The burden of disease in Spain: Results from the Global Burden of Disease 2016. Med Clin (Barc) 151:171-190.

https://doi.org/10.1016/j.medcli.2018.05.011

21. Donnelly S (2020) The Elderly \& COVID-19: Cocooning or Culling: - the choice is ours. QJM. https://doi.org/10.1093/qjmed/hcaa145

\section{Figures}

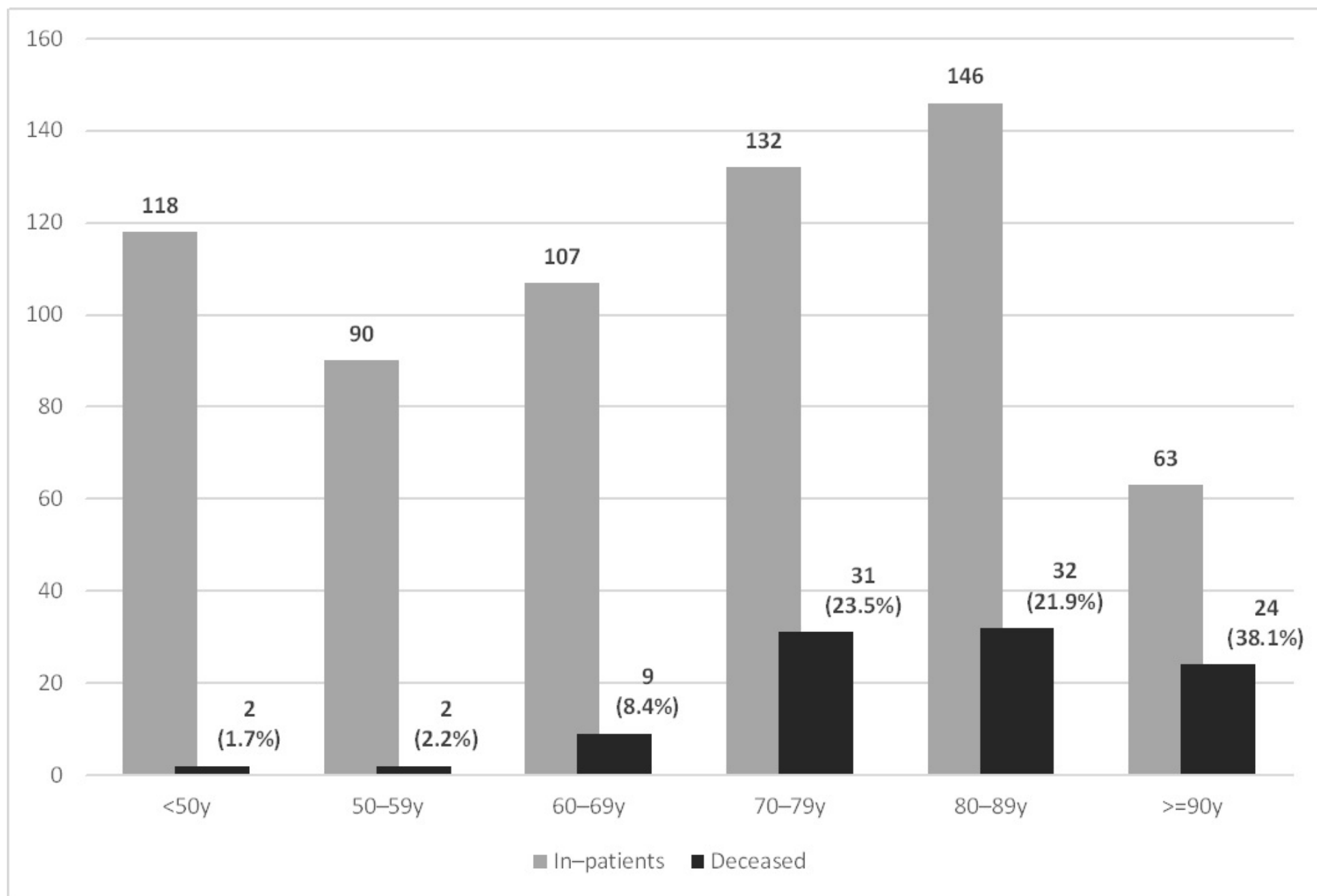

\section{Figure 1}

Number of inpatients and mortality rate according to age. 


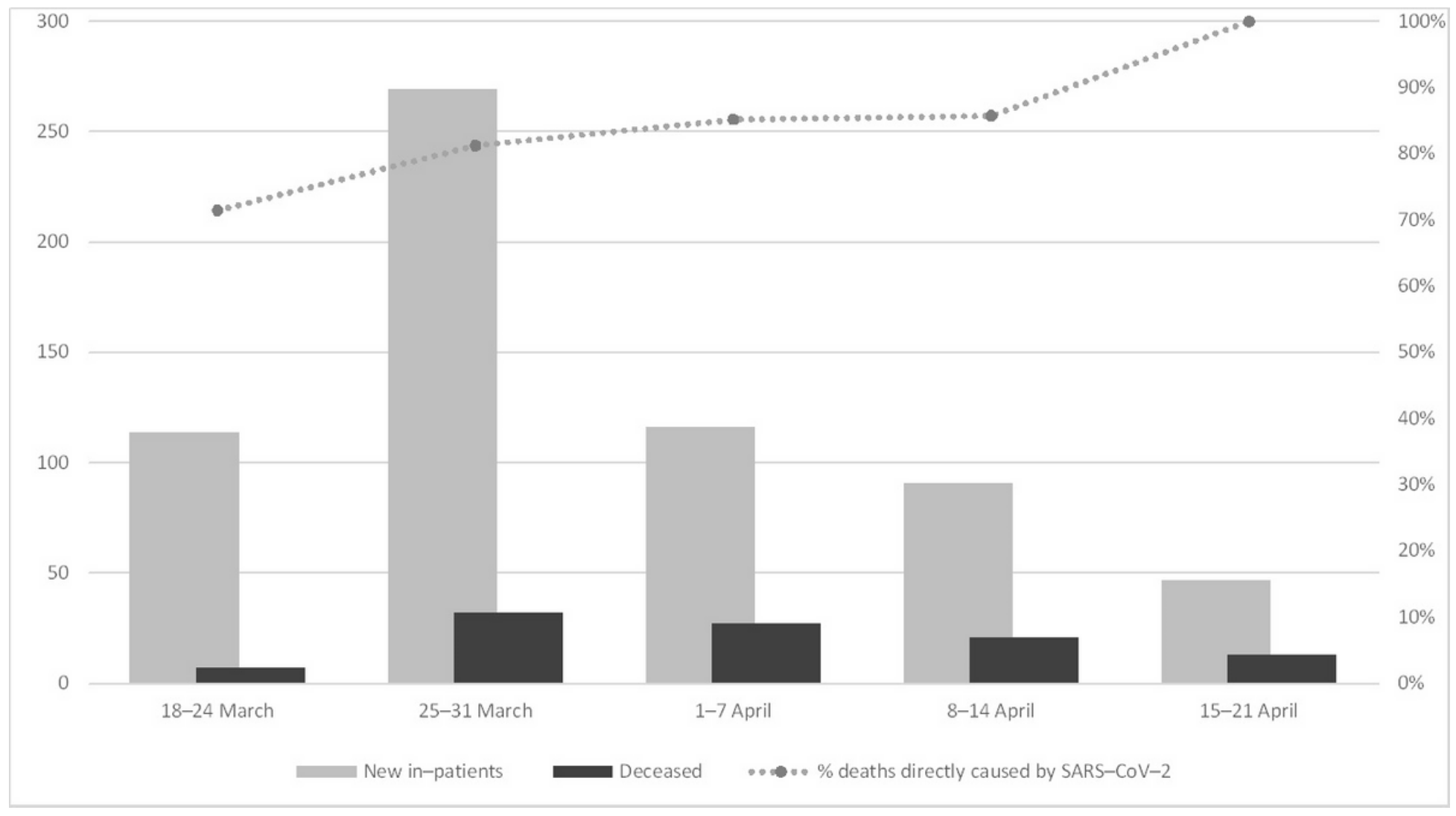

\section{Figure 2}

Evolution of the proportion of deaths caused directly by SARS-CoV-2 infection.

\section{Supplementary Files}

This is a list of supplementary files associated with this preprint. Click to download.

- GraphicalAbrast.jpg 